\title{
Rhythmicity of Prefrontal Local Field Potentials after Nucleus Basalis Stimulation
}

\author{
Balbir Singh, ${ }^{1}{ }^{\circ}$ Xue-Lian $\mathrm{Qi}^{2}{ }^{2}$ David T. Blake, ${ }^{3}$ and Christos Constantinidis ${ }^{1,4,5}$
}

\author{
https://doi.org/10.1523/ENEURO.0380-21.2022
}

${ }^{1}$ Department of Biomedical Engineering, Vanderbilt University, Nashville, TN 37235, 2Department of Neurobiology and Anatomy, Wake Forest School of Medicine, Winston-Salem, NC 27157, ${ }^{3}$ Department of Neuroscience and

Regenerative Medicine, Medical College of Georgia, Augusta University, Augusta, GA 30912, ${ }^{4}$ Neuroscience Program, Vanderbilt University, Nashville, TN 37235, and ${ }^{5}$ Department of Ophthalmology and Visual Sciences, Vanderbilt University Medical Center, Nashville, TN 37232

\begin{abstract}
The action of acetylcholine in the cortex is critical for cognitive functions and cholinergic drugs can improve functions such as attention and working memory. An alternative means of enhancing cholinergic neuromodulation in primates is the intermittent electrical stimulation of the cortical source of acetylcholine, the nucleus basalis (NB) of Meynert. NB stimulation generally increases firing rate of neurons in the prefrontal cortex, however its effects on single neurons are diverse and complex. We sought to understand how NB stimulation affects global measures of neural activity by recording and analyzing local field potentials (LFPs) in monkeys as they performed working memory tasks. NB stimulation primarily decreased power in the alpha frequency range during the delay interval of working memory tasks. The effect was consistent across variants of the task. No consistent modulation in the delay interval of the task was observed in the gamma frequency range, which has previously been implicated in the maintenance of working memory. Our results reveal global effects of cholinergic neuromodulation via deep brain stimulation, an emerging intervention for the improvement of cognitive function.
\end{abstract}

Key words: acetylcholine; basal forebrain; LFP; monkey; neurophysiology; working memory

\section{Significance Statement}

Stimulation of the endogenous source of acetylcholine in the cerebral cortex, the nucleus basalis (NB) of Meynert has shown promise as an alternative to cholinergic drugs, which are frontline medications for the treatment of Alzheimer's disease and age-related dementia. The neural mechanisms through which stimulation may improve cognitive function are incompletely understood. Here, we investigate the effects of NB stimulation in the prefrontal cortex by examining the power of the local field potentials (LFPs) during execution of working memory tasks.

\section{Introduction}

The forebrain cholinergic system is essential for the performance of cognitive functions (Sarter et al., 2016; Galvin et al., 2018). Cognitive decline in age-related dementia and Alzheimer's disease occurs in parallel with cholinergic degeneration (Terry and Buccafusco, 2003; Ballinger et al., 2016). Animal models similarly exhibit profound cognitive deficits following cholinergic depletion (Chudasama

\footnotetext{
Received September 17, 2021; accepted January 11, 2022; First published January 20, 2022.

The authors declare no competing financial interests.
}

et al., 2004; Croxson et al., 2011). Conversely, cholinergic agonists and cholinesterase inhibitors can improve cognitive function (Birks and Harvey, 2018), as can stimulation of the endogenous source of acetylcholine in the cerebral cortex, the nucleus basalis (NB) of Meynert (Mesulam et al., 1983). Recent experiments in non-human primates have demonstrated the feasibility of cholinergic deep brain stimulation in improving cognitive function, in young

Author contributions: X.-L.Q., D.T.B., and C.C. designed research; X.-L.Q., D.T.B., and C.C. performed research; B.S., X.-L.Q., and C.C. analyzed data; C.C. wrote the paper. 
adult animals (Blake et al., 2017; Liu et al., 2017, 2018). Pilot studies have also been conducted in humans, with encouraging results in terms of the safety and tolerance of the procedure, although no dramatic cognitive improvements have been demonstrated so far (Freund et al., 2009; Kuhn et al., 2015; Hardenacke et al., 2016). Substantial obstacles remain for clinical translation of cholinergic deep brain stimulation, including that considerable degeneration of the basal forebrain may have already occurred by the time of a clinical dementia diagnosis (Nazmuddin et al., 2021).

Investigating the effects of NB stimulation on neuronal activity is essential for understanding its mechanism of action and optimizing its effectiveness (Subramaniam et al., 2021). NB stimulation affects neuronal activity in the prefrontal cortex during performance of working memory tasks (Qi et al., 2021). Reported results generally indicated an increase in mean firing rate of prefrontal neurons; however, the effects were considerably diverse, including a substantial minority of neurons being suppressed by stimulation. The effects of stimulation on measures of population activity and rhythmicity such as the local field potential (LFP) have not been known. We were motivated therefore to investigate effects of NB stimulation by examining the power of the prefrontal LFP.

LFP represents summation of ionic currents in a limited cortical volume in the order of $0.1-$ to $0.2-\mathrm{mm}$ radius (Buzsaki, 2004; Kajikawa and Schroeder, 2011). During presentation of stimuli, correlated bottom-up inputs can serve to synchronize population neuronal spiking, and phases of synchronized excitation by pyramidal neurons followed by inhibition by interneurons can thus produce rhythmicity in the field potentials (Fries, 2009). LFP rhythmicity in the $\gamma$ frequency range in particular is well known to emerge in the delay period of working memory tasks and to be tuned for information held in memory, e.g., spatial location of a remembered cue (Pesaran et al., 2002; Holmes et al., 2018). $\gamma$ Oscillations are highly detectible in other extracellular field recordings (such as EEG or MEG) and are thus a reliable marker of underlying cognitive processes impacting neural circuit interactions (Uhlhaas and Singer, 2011; Roux and Uhlhaas, 2014). Our current study investigated the impact of NB stimulation on LFP potential measures of rhythmic firing during performance of working memory tasks.

\section{Materials and Methods}

Two male, rhesus monkeys (Macaca mulatta) aged 1213 years old, and weighing $7-10 \mathrm{~kg}$ were used in this

This work was supported by National Institutes of Health Grants R01 MH097695 and RF1 AG060754.

Acknowledgments. We thank Zhengyang Wang for valuable comments on this manuscript.

Correspondence should be addressed to Christos Constantinidis at christos.constantinidis.1@vanderbilt.edu.

https://doi.org/10.1523/ENEURO.0380-21.2022

Copyright $@ 2022$ Singh et al.

This is an open-access article distributed under the terms of the Creative Commons Attribution 4.0 International license, which permits unrestricted use, distribution and reproduction in any medium provided that the original work is properly attributed. study. All experimental procedures followed guidelines by the United States Public Health Service Policy on Humane Care and Use of Laboratory Animals and the National Research Council's Guide for the Care and Use of Laboratory Animals and were reviewed and approved by the Institutional Animal Care and Use Committee (IACUC).

\section{Surgery and neurophysiology}

A $20 \mathrm{~mm}$-diameter recording cylinder was implanted over areas $8 a$ and 46 (Fig. $1 A$ ), residing in the posterior aspect of the dorsolateral prefrontal cortex (Riley et al., 2017). Recordings of single units and LFPs were obtained with arrays of epoxylite-coated tungsten microelectrodes with a $250-\mu \mathrm{m}$ diameter and 1- to $4-\mathrm{M} \Omega$ impedance at $1 \mathrm{kHz}$ (FHC). Typically, three to four electrodes were lowered into the cortex in a single recording session. The signal was split and differentially filtered via hardware means for single units $(500 \mathrm{~Hz}$ to $8 \mathrm{kHz})$ and LFPs $(0.5-100 \mathrm{~Hz})$, and LFP data were captured at a $500-\mathrm{Hz}$ sampling rate. Deep brain electrodes reaching the NB were implanted unilaterally (in the left, and in the right hemisphere, respectively for the two animals) at 8 $\mathrm{mm}$ lateral, $16 \mathrm{~mm}$ anterior interaural, and $29 \mathrm{~mm}$ below the cortical surface in a vertical penetration. Stimulation electrodes were fabricated in-house based on published specifications (McCairn and Turner, 2009). Conductors were $50 \mu \mathrm{m} \mathrm{Pt} / \mathrm{lr}$, Teflon-insulated wire (AM Systems). The last $0.7 \mathrm{~mm}$ of insulation was stripped to achieve impedances of $5-10 \mathrm{k} \Omega$ at $1 \mathrm{kHz}$. Stimulation pulses were created by an isolated pulse stimulator (Model 2100, AM Systems), which was controlled by custom programed software, written on the MATLAB computational environment (MathWorks). Stimulation was delivered with biphasic, negative first, unipolar 200- $\mu \mathrm{A}$ pulses with $100 \mu$ s per phase, and $80-\mathrm{Hz}$ pulses were delivered for $15 \mathrm{~s}$.

\section{Behavioral tasks}

The monkeys faced a computer monitor $60 \mathrm{~cm}$ away in a dark room with their head fixed. Eye position was sampled at $240 \mathrm{~Hz}$, digitized, and recorded with an infrared eye position tracking system (model RK-716; ISCAN). The visual stimulus presentation and behavior monitoring were controlled by in-house software (Meyer and Constantinidis, 2005) implemented in MATLAB. The tasks used in the present study were variations of the oculomotor delayed response (ODR) task, but involving two visual stimuli appearing in sequence. The tasks required the monkey to remember and make an eye movement to the location of either the first or the second visual stimulus depending on the color of fixation point (Fig. 2A). After the animals fixated at a white/blue fixation point located at the center of the monitor for $1 \mathrm{~s}$, two white squares $\left(1.5^{\circ}\right.$ in size) were displayed sequentially for $0.5 \mathrm{~s}$, with a $1-\mathrm{s}$ intervening delay period. The first visual stimulus was displayed at one of eight possible locations arranged along a circular ring of $12^{\circ}$ eccentricity, with a $45^{\circ}$ angular separation between neighboring 


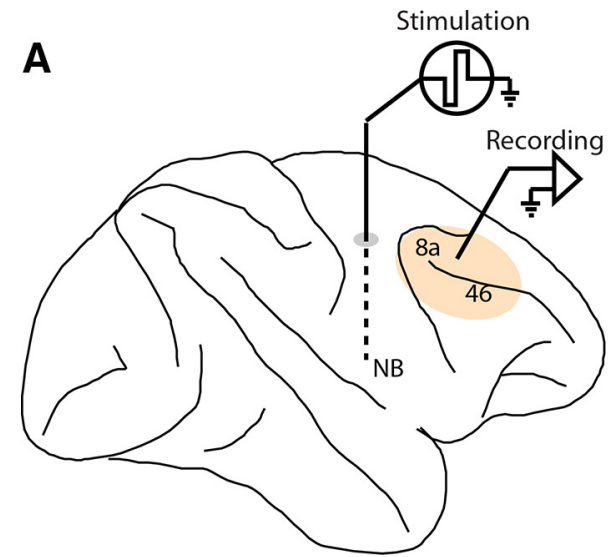

B

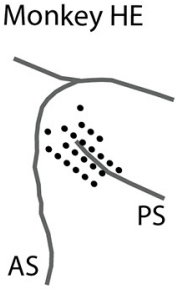

$10 \mathrm{~mm}$
Monkey GR

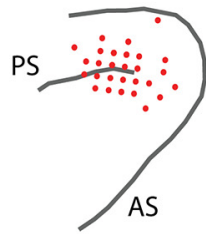

Figure 1. Stimulation and recording areas. $\boldsymbol{A}$, Schematic diagram of the monkey brain, with the cortical region sampled with neurophysiological recordings and relative location of the stimulation recording indicated. $\boldsymbol{B}$, Penetration maps of where neurons and LFPs were recorded in two animal subjects (HE and GR). PS, principal sulcus; AS, arcuate sulcus.

visual stimuli. The monkeys were trained with stimulus appearances at every possible location, however, during recordings the first stimulus appeared at one of these eight locations and its diametric location. We generally attempted to place one of the two stimuli in the receptive field of a neuron recorded from our electrodes, however recordings were performed with multiple electrodes and the stimulus may have appeared at any location of a neuron's receptive field. This first stimulus followed by a delay period and then a second visual stimulus, which was displaced $0^{\circ}, 45^{\circ}, 90^{\circ}$, or $180^{\circ}$ relative to the first.
Two additional, "null" conditions were included, in which either the first or second stimulus presentation was omitted, so that there were 10 trial types in total, and these were used with equal frequency. After a second delay period of $1 \mathrm{~s}$, the monkeys were required to saccade to the location of the first visual stimulus if the fixation point was white in color (remember-first condition), and to the location of the second visual stimulus if the fixation point was blue (remember-second condition). The monkeys were rewarded with juice after making a correct saccade. Deviating gaze beyond a $3^{\circ}$ radius fixation window

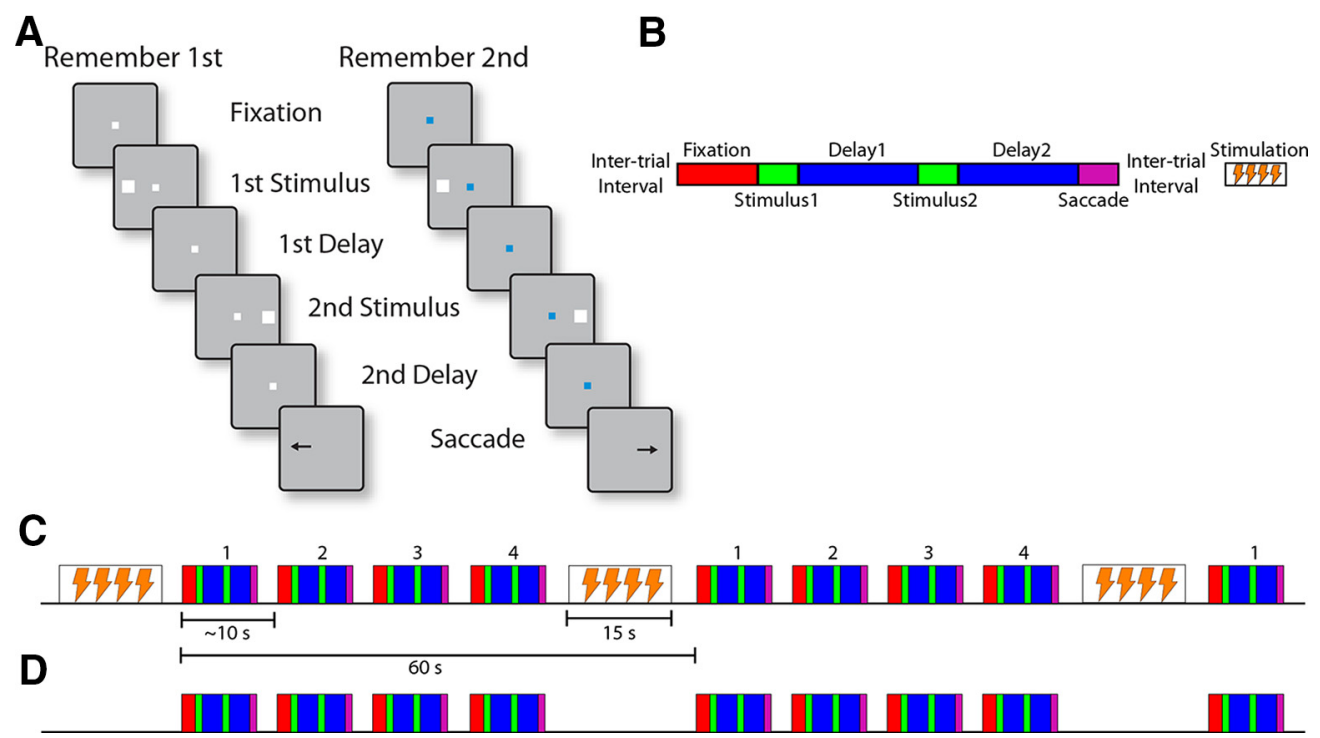

Figure 2. Behavioral task. $\boldsymbol{A}$, Successive frames illustrate the sequence of events in the behavioral task. Depending on the white or blue color of the fixation point, the monkey has to remember either the first or the second of two visual stimuli presented in sequence, respectively. At the end of the trial, the fixation point turns off and the monkey needs to perform an eye movement toward the remembered location of the visual stimulus. $\boldsymbol{B}$, Schematic diagram of a single trial of the task. Blocks represent the successive events in the task: fixation, first visual stimulus presentation, first delay period, second visual stimulus presentation, second delay period, and saccade. Successive trials are separated by intertrial intervals. NB stimulation, when delivered, always occurs during the intertrial interval. $\boldsymbol{C}$, Sequence of trials during a stimulation block, in a compressed time scale, relative to panel $\boldsymbol{B}$. Successive trials, labeled $1-4$, each lasting $\sim 10 \mathrm{~s}$, are followed by $15 \mathrm{~s}$ of stimulation. $\boldsymbol{D}$, Sequence of trials during a control (no-stimulation) block. The trials are arranged in the same fashion as in the stimulation block, including an extended intertrial interval every $60 \mathrm{~s}$, during which however no stimulation is applied (sham). 
led to the immediate termination of the trial without reward. To minimize the uncertainty about the visual stimulus to be remembered, the remember-first and remember-second conditions were presented in blocks of trials. The animal was required to perform 10 correct trials of the remember-first task, before the task alternated to the remember-second condition. During sessions of NB stimulation that were delivered in block mode (trials with and without stimulation collected during the same daily session), $\sim 60$ trials were collected for each block, which therefore involved six alterations between the remember-first and remember-second rule. A control block of trials was acquired first, followed by a stimulation block of trials. Intermittent stimulation was applied for $15 \mathrm{~s}$ at 80 pulses per second, followed by $\sim 45 \mathrm{~s}$ with no stimulation (Fig. 2B). Stimulation was applied in the inter-trial interval, after a trial had completed, and a new trial began after stimulation had elapsed.

\section{LFP analysis}

We used the FieldTrip toolbox (Oostenveld et al., 2011) for preprocessing analysis and Chronux package (Bokil et al., 2010) for time-frequency analysis. For power analysis of LFP signals from the recording electrodes, a bandpass filter $(0.5-100 \mathrm{~Hz})$ was used. We removed line power $(60 \mathrm{~Hz})$ and other artifacts from each electrode and trial in the LFP signal, if present. We then used a multi-taper method to perform a power spectrum analysis of LFP. Time-resolved plots of power (spectrograms) were constructed and plotted after subtracting the mean power of the baseline period at each frequency. The baseline period was defined to be last $1 \mathrm{~s}$ in the inter-trial interval apart from the trials in which the NB stimulation was delivered (time $>5 \mathrm{~s}$ after the onset of the fixation point). This occurred at least $1 \mathrm{~s}$ after the end of the trial and delivery of reward, at a time when the monkey was free to make eye movements, before the appearance of the next stimulus. Alternatively, we used the $1 \mathrm{~s}$ of fixation before the cue presentation as baseline for some analysis. We then compared the LFP power at each frequency between the control and simulation conditions. We also analyzed the LFP power at different frequency bands defined as $\alpha(8-14 \mathrm{~Hz}), \beta$ (20$35 \mathrm{~Hz})$, and $\gamma(45-100 \mathrm{~Hz})$ after subtracting the mean power. We obtained estimates of the variability of these measures by using a bootstrap technique. We randomly selected with replacement $75 \%$ of trials from each of electrode and repeated the power spectrum estimation 1000 times. We compared power between different conditions (control vs NB stimulation; remember-first vs remember-second; correct vs error trials) on a time point by point fashion, averaging power over a 250-ms window, with a step of $100 \mathrm{~ms}$. We then used a permutation test that randomized the label of trials across conditions while preserving information about which electrode each trial originated. This was evaluated at the $\alpha=0.01$ significance level with a twotailed test.

\section{Results}

Two monkeys were implanted with unilateral electrodes targeting the NB to determine the effects of stimulation on behavioral performance and neural activity. Electrode placement was guided by MR imaging (Fig. $1 A)$. Neurophysiological recordings were then obtained from areas $8 a$ and 46 of the dorsolateral prefrontal cortex (Fig. 1B). The monkeys performed a task requiring them to view two stimuli presented in sequence and make an eye movement to the remembered location of either the first or second of them (Fig. 2A). A white fixation point instructed the monkeys to remember the first stimulus; a blue fixation point required them to remember the second stimulus. The two stimuli could appear at any of eight possible locations, presented in a randomized order. When NB stimulation was delivered, it was applied during an inter-trial interval between two trials for $15 \mathrm{~s}$, at a frequency of 80 pulses per second (Fig. 2B,C). The monkeys performed consecutive trials for $45 \mathrm{~s}$ (typically four to five completed trials), without NB stimulation. At the end of the trial that exceed the 45-s threshold, NB stimulation was applied, and the cycle was repeated. Blocks of trials were obtained first under control conditions and then under NB stimulation, in daily sessions.

\section{Effects of NB stimulation on LFP spectral power}

We recorded LFPs from electrodes in the prefrontal cortex (areas $8 \mathrm{a}$ and 46) from which single-unit recordings were also obtained. The resulting dataset consisted of 7846 trials from 126 LFP sites in control condition and 6201 trials from 132 LFP sites in NB stimulation condition. Synchronization of LFP power in the $\gamma$ frequency band has been proposed as the critical neural correlate of working memory maintenance (Lundqvist et al., 2018). We therefore examined the LFPs recorded from the prefrontal cortex, in the $\alpha$ (8-14), $\beta$ (20-35), and $\gamma(45-100)$ frequency bands, as these bands were defined in previous studies supporting the latter theory (Lundqvist et al., 2016), and tested whether the effects of NB stimulation increased $\gamma$ power during the delay periods of the task.

We calculated average spectral power during the course of the trial, after subtracting the mean power from a baseline period in the intertrial period. Data analyzed in this fashion allowed us to determine spectral power changes driven by task events in each trial and how these differed between conditions (shown in Fig. $3 A, B$ ). This analysis averaged all conditions, task types, and stimulus locations, including catch trials. The caveat in this analysis is that changes between stimulation and control condition that may be present after the end of the trial and delivery of reward may not be detectable, and we therefore omit comparisons at the end of the trial. The most salient effect of NB stimulation plotted was a decrease in the $\alpha$ frequency range (Fig. $3 C, D$ ). The effect was most pronounced during the stimulus presentations and the first delay period. We compared power in the same electrodes, and the same behavioral sessions, when NB stimulation was applied and when it was not. Stars in Figure $3 D$ indicate individual time points where the difference 

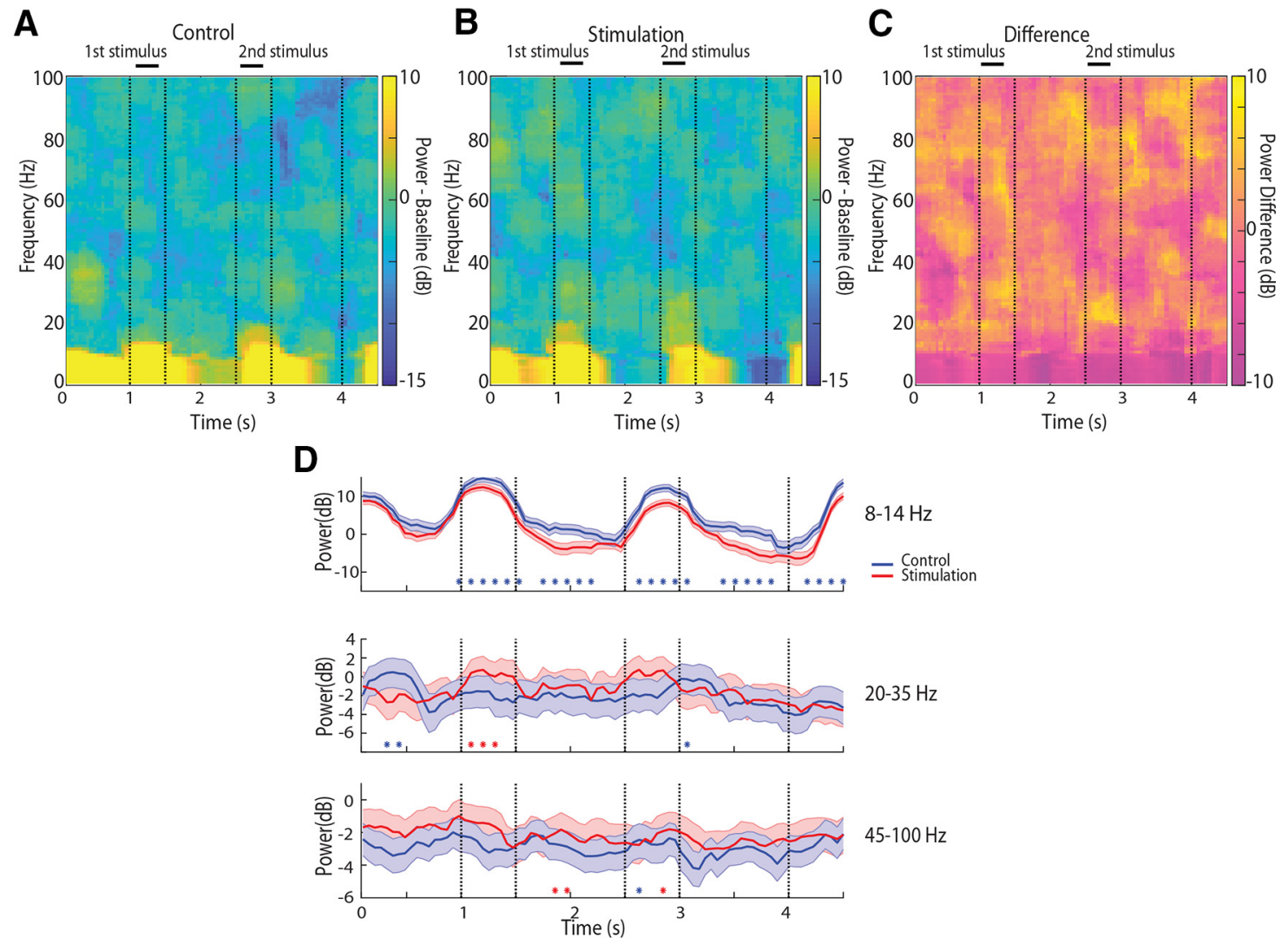

Figure 3. Time-resolved power of LFPs. A, Spectrograms of LFPs recorded from the prefrontal cortex during the control condition, after subtracting the mean power of the baseline period at each frequency band $(n=7846$ trials for the control and 6201 for the stimulation condition). Horizontal lines indicate time of stimulus presentations. $\boldsymbol{B}$, Mean power after subtracting the baseline during the stimulation condition. $\boldsymbol{C}$, Difference in power between stimulation and control. Positive values indicate higher power in stimulation condition. $\boldsymbol{D}$, Time course of spectral power, after subtracting the baseline, in the $\alpha, \beta$, and $\gamma$ frequency bands for the control and stimulation conditions. Shaded regions around each line represent $95 \%$ confidence intervals, estimated with a bootstrap method.

statistical significance based on a permutation test evaluated at the 0.01 significance level.

We also relied on a general linear model analysis, computing spectral power across each task epoch, in each trial, and comparing this power between epochs, and stimulation conditions. This analysis confirmed a highly significant main effect of NB stimulation (two-way ANOVA, $F_{(1,70225)}=335.7$, $p=3.7 \times 10^{-79}$ ). $\alpha$ Power computed in this was significantly lower under NB stimulation than control for each of the task epoch conditions (Tukey's post hoc test, $p<0.05$ for each of the fixation, stimulus1, delay1, stimulus2, delay2 periods). This decrease in $\alpha$ power was also evident in both subjects, when processed separately (Fig. 4).

NB stimulation produced more subtle effects in the $\beta$ frequency band. $\beta$ Power appeared elevated during the stimulus presentation periods, with several time points in that interval indicating a significant difference between conditions (Fig. 3D). However, the effect was driven by a single subject (Fig. 2F); for the second subject, the opposite trend of decreased $\beta$ power with NB stimulation was evident. Averaging power across each task epoch revealed no significant difference between power in the control and NB stimulation condition in the $\beta$ frequency band (two-way ANOVA, $F_{(1,70225)}=0.32, p=0.57$ for main effect of NB stimulation). No individual task epoch exhibited a significant difference in $\beta$ power either (Tukey's post hoc test, $p>0.05$ for each of the fixation, stimulus1, delay1, stimulus2, and delay2 periods).

An increase in $\gamma$ band power was also evident with NB stimulation. Individual time points reached significance in the delay and second stimulus period (Fig. $3 D$ ). Averaging power across each task epoch revealed a significant increase of $\gamma$ power in the NB stimulation condition (twoway ANOVA, $\left.F_{(1,70225)}=73.37, p=1.09 \times 10^{-17}\right)$. This difference reached statistical significance in the fixation, stimulus1 and delay1 epochs (Tukey's post hoc test, $\mathrm{p}<0.05$ ).

An important consideration in this analysis was the choice of baseline. We relied on the inter-trial interval, a period over which no stimuli were presented and animals were unconstrained to generate eye movements, which could potentially skew the results. We therefore repeated the analysis using the $1 \mathrm{~s}$ of the fixation period as baseline, instead (Fig. 4C). Spectral power relative to the fixation period produced a near identical pattern of significant differences between control and NB stimulation conditions in the $\alpha$ band (Fig. 4C, top). No systematic differences between conditions were present in the $\beta$ frequency band (Fig. $4 C$, middle). $\gamma$ Band differences were most affected by this choice of baseline, however NB stimulation 

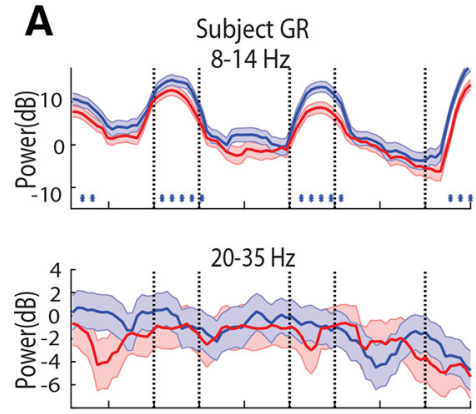

$45-100 \mathrm{~Hz}$

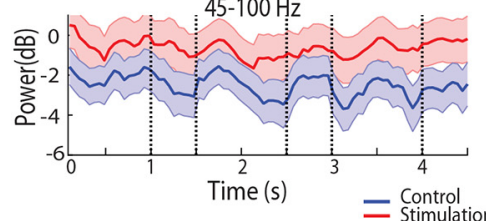

B
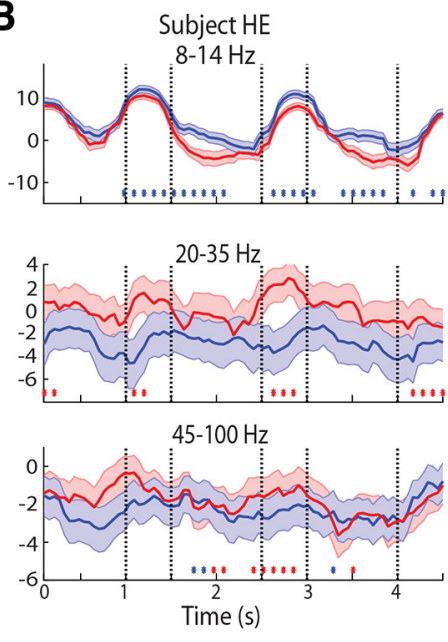
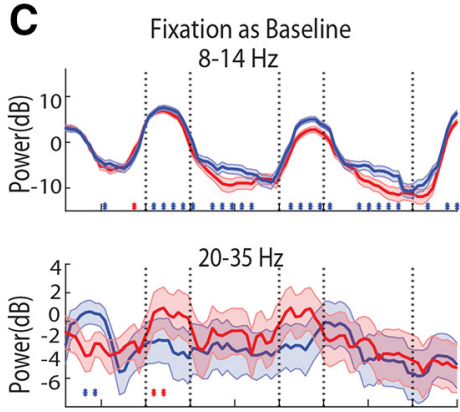

$45-100 \mathrm{~Hz}$

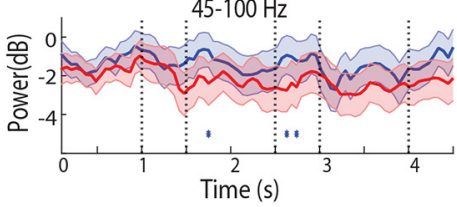

Figure 4. Spectral power for different subjects and baseline conditions. $\boldsymbol{A}$, Time course of spectral power, after subtracting the baseline, in the $\alpha, \beta$, and $\gamma$ frequency bands for the control and stimulation conditions plotting results from subject GR only. $\boldsymbol{B}$, As in $\boldsymbol{A}$, plotting results from subject HE only. $\boldsymbol{C}$, Time course of spectral power, after subtracting the fixation period as baseline. Stars represent significant difference between the control and NB stimulation conditions, evaluated with a permutation test, at the 0.01 significance level.

produced even lower $\gamma$ band power during the delay period (Fig. 4C, bottom). In other words, NB stimulation increases $\gamma$ power from the fixation period onward, rather than specifically for the delay period of the task, so that using the fixation period as a baseline makes no increase in $\gamma$ power apparent during the course of the trial.

Another important consideration for our analysis was the choice of spectral bands. It is possible that our a priori selection of the three frequency bands where we averaged spectral power did not correspond well to frequency zones that were modulated by task factors. For this reason, we repeated our statistical analysis across the whole time-frequency space and identified clusters with a permutation-based approach (Maris and Oostenveld, 2007). The results are shown in Figure 5. The analysis revealed that our definition of the $\alpha$ band $(8-14 \mathrm{~Hz})$ corresponded well with a zone of spectral power that was reduced by NB stimulation between 8 and $18 \mathrm{~Hz}$ (Fig. $5 A, B$ ), which additionally did not overlap with our definition of $\beta$ band. Similarly, a spectral zone of enhanced NB stimulation was observed between 25 and $40 \mathrm{~Hz}$, which mostly coincided with our $\beta$ band definition $(20-35 \mathrm{~Hz})$. No consistent effect was observed in the $\gamma$ frequency band. Performing this analysis separately in the two animals confirmed our conclusions that $\alpha$ power modulation was most consistent between the two animals; effects in the $\beta$ and $\gamma$ frequency moved essentially in opposite directions (Fig. 5C,D).

\section{LFP spectral power across tasks}

We next wished to test the effects of NB stimulation in the context of the two variants of the task the monkeys performed, requiring them to remember either the first or second stimulus displayed and how NB stimulation affected each. The remember-second task was generally more difficult for the monkeys, possibly because they learnt the remember-first task earlier (Qi et al., 2021). We wished to test therefore whether $\gamma$ power, in particular, differed systematically between tasks. This was indeed the case. The spectrogram constructed separately for the two tasks is shown in Figure 6, again averaging all stimulus conditions. In the control condition, power in the $\gamma$ frequency band was generally higher in the remembersecond task compared with the remember-first task (Figs. $6 A, 7 A$ ). This difference was maximal and reached statistical significance late in the period of the first stimulus presentation and early in the delay period (Fig. 7A).

We also sought to compare power between tasks in the $\alpha$ frequency range. $\alpha$ Power has been implicated in allocation of attention and filtering of distractors (Klimesch, 2012), the timing of which differed in our two tasks. Indeed, we observed that early in the trial, during the fixation period and first stimulus presentation, $\alpha$ power was higher in the remember first than the remember second task (Fig. 7A, cyan stars in top panel). This pattern reversed, however, during the second stimulus presentation, which constituted a distractor for the remember-first task but the stimulus to be maintained in memory for the remembersecond task (Fig. 7A, purple stars in top panel). Using a three-way ANOVA model with epoch, NB stimulation, and task (remember-first/remember-second) factors revealed a significant main effect of task $\left(F_{(1,70219)}=9.79, p=0.002\right)$ as well as an epoch $\times$ task interaction $\left(F_{(4,70219)}=4.97\right.$, $p=5 \times 10^{-4}$ ).

NB stimulation tended to reduce the differences between tasks. Both the elevated $\gamma$-band power in the remember-second task, and the alternating pattern of $\alpha$ power during the two stimulus presentations was greatly reduced under NB stimulation (Fig. 7B). Only a handful of time points now exhibited a significant difference in the $\gamma$ band, based on the same permutation test, evaluated at 
A
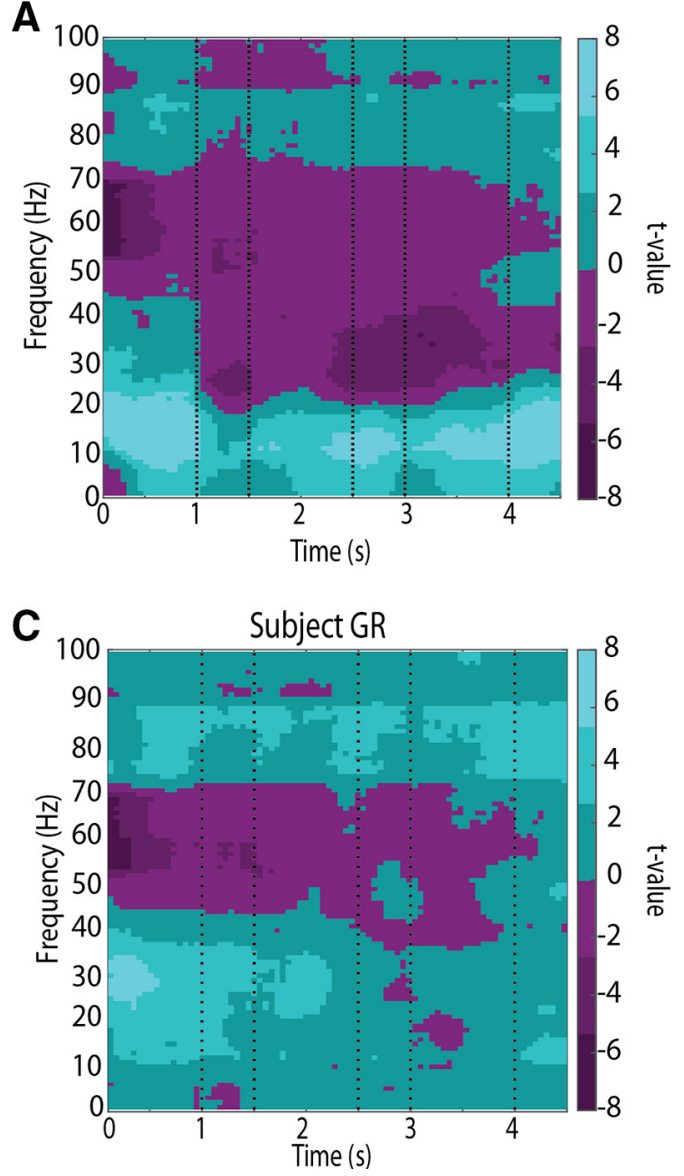

B
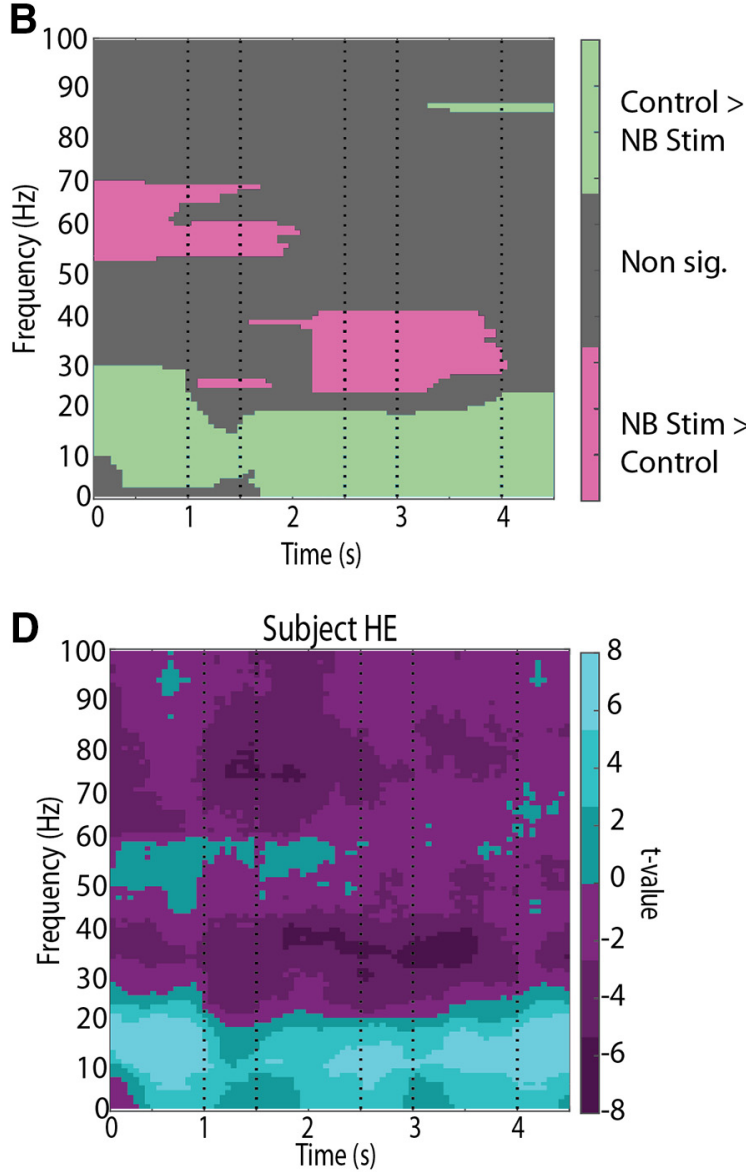

Figure 5. Cluster-based spectral analysis. $\boldsymbol{A}$, Color plot represents two-sample $t$ test $t$ values comparing the time point-by-time point LFP spectral power in the NB stimulation relative to the control condition. $\boldsymbol{B}$, Clusters of power difference between the control and NB stimulation condition, based on a permutation test that was based on $t$ values shown in $\boldsymbol{A}$. Color plot at each point cluster represents the bootstrapped $p$ value. $\boldsymbol{C}$, As in $\boldsymbol{A}$, plotting results from subject $\mathrm{GR}$ only. $\boldsymbol{D}$, As in $\boldsymbol{A}$, plotting results from subject $\mathrm{HE}$ only.

the 0.01 significance level (Fig. 7B, lower panel). The overall suppressive main effect of NB stimulation was also highly significant in the three-way ANOVA model $\left(F_{(1,70219)}=72.82, p=1.4 \times 10^{-17}\right)$. In the $\alpha$ band, NB stimulation decreased power in the first stimulus presentation period and increased it in the second, resulting in little difference between tasks. This opposing effect was evident as a significant NB stimulation $\times$ task interaction term in the threeway ANOVA model $\left(F_{(1,70219)}=23.6, p=1.2 \times 10^{-6}\right)$.

\section{Spectral power in error and catch trials}

Differences in power between the control and NB stimulation as well as between task conditions do not necessarily imply that these reflect differences in performance of the task. To gain insight on the power distribution that characterized successful task performance, we compared LFP power between correct and error trials (Fig. 8). We averaged responses from all error trials, excluding breaks in fixation, which resulted in premature termination of the trial. The most frequent type of error was a saccade toward the incorrect stimulus (second stimulus in the remember-first task, or first stimulus in the remember-second task).
We first sought to test whether $\gamma$ power decreased in error trials relative to correct, as would be predicted by theories that consider $\gamma$ rhythmicity the critical neural variable of working memory maintenance. This did not prove to be the case. If anything, error trials were associated with higher power in the $\gamma$ frequency range (Fig. 8). When $\gamma$ power was averaged within each epoch, the effect of correct or error trial outcome was highly significant in a three-way ANOVA model with epoch, NB stimulation, and correct/error factors $\left(F_{(1,86964)}=163.8, p=1.8\right.$ $\left.\times 10^{-37}\right)$. This difference between correct and error trials was observed for both the control (Fig. 8A) and NB stimulation conditions (Fig. 8B). Had we relied on the fixation interval as our baseline (as in Fig. 4C), this analysis would yield essentially identical $\gamma$ power in correct and error trials.

A decrease in $\alpha$ power was also evident in error trials (Fig. 8A). The main effect of trial outcome was highly significant in the three-way ANOVA model $\left(F_{(1,86964)}=91.3\right.$, $\left.p=1.3 \times 10^{-21}\right)$. NB stimulation had a similar effect on correct and error trials, evidenced by an absence of a significant interaction term in the model $\left(F_{(1,86964)}=1.78\right.$, $p=0.18$ ). 
A

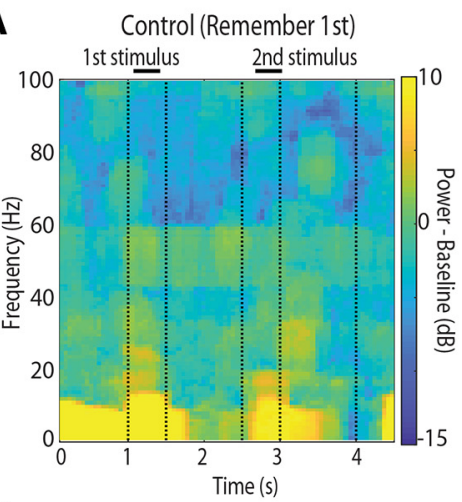

D

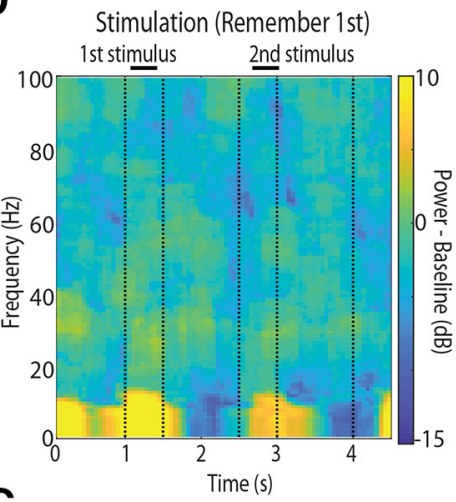

G

Stimulation-Control (Remember 1st)

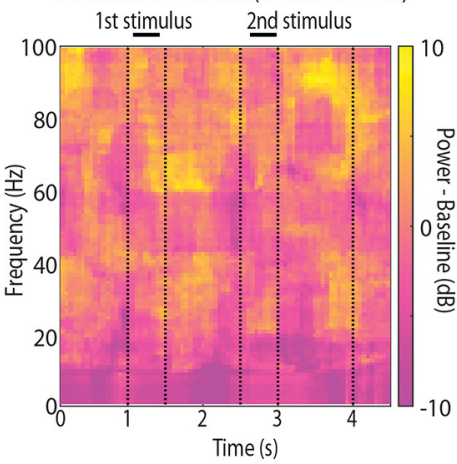

B Control (Remember 2nd)

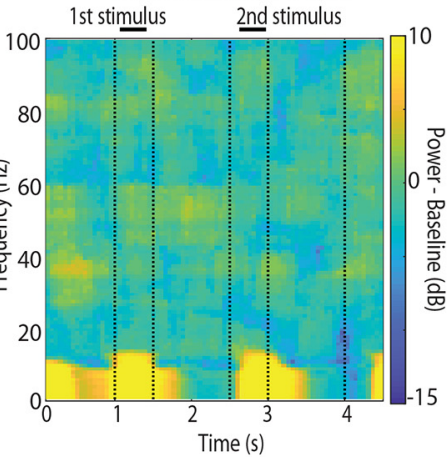

E Stimulation (Remember 2nd)

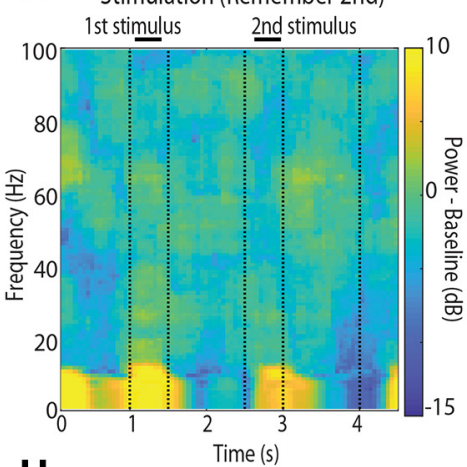

H

Stimulation-Control (Remember 2nd)

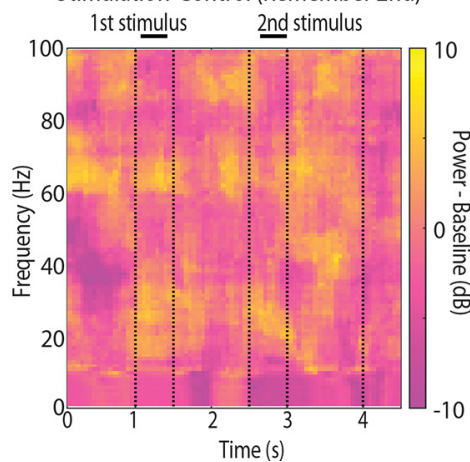

C Remember 2nd-Remember 1st

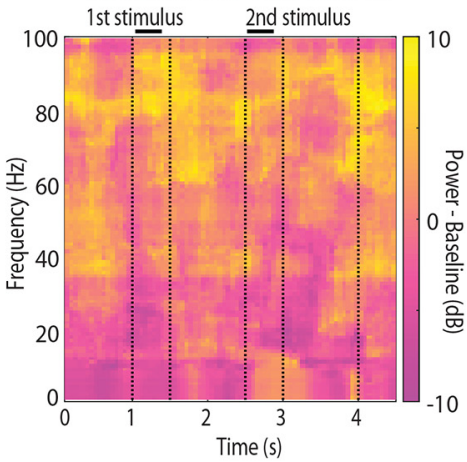

F Remember 2nd -Remember 1st 1st stimulus 2nd stimulus

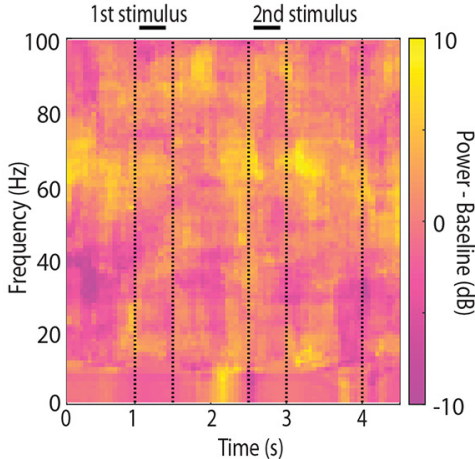

Figure 6. Power spectrum for remember-first and remember-second task. $\boldsymbol{A}$, Spectral power of LFPs recorded from the prefrontal cortex during the control condition in the remember-first task, after subtracting the mean power of the baseline fixation period at each frequency band ( $n=3961$ trials). $\boldsymbol{B}$, As in $\boldsymbol{A}$, for the remember-second task ( $n=3885$ trials). $\boldsymbol{C}$, Difference in power between remember-first and remember-second tasks in the control condition. $\boldsymbol{D}$, As in a $\boldsymbol{A}$, for the NB stimulation condition ( $n=3143$ trials). $\boldsymbol{E}$, As in $\boldsymbol{B}$, for the NB stimulation condition ( $n=3058$ trials). $\boldsymbol{F}$, Difference in power between remember-first and remember-second tasks in the NB stimulation condition. $\boldsymbol{G}$, Difference in power between control and NB stimulation in the remember-first task. $\boldsymbol{H}$, Difference in power between control and NB stimulation in the remember-second task.

We additionally examined spectral power in catch trials, containing no first stimulus. The timing of stimulus presentation was fixed in our task, so that the first stimulus was presented after $1 \mathrm{~s}$ of fixation, which presumably the monkeys could time, however, in $10 \%$ of the trials the first stimulus was omitted. A second stimulus was still presented at its expected time, in these trials. NB stimulus has been shown to induce "phantom" peaks of firing rate increases during the anticipated time of the missing stimulus presentation (Qi et al., 2021) and we were interested to test whether spectral power would deviate in control and NB stimulation conditions. Indeed, we found an increase in power under NB stimulation, which was evident in all three spectral bands we examined (Fig. 9). Stars in Figure 9 indicate individual time points where the difference statistical significance based on a permutation test evaluated at the 0.01 significance level, indicating most prominently an increase during the cue period. In the $\alpha$ frequency band, despite this transient increase in power under NB stimulation, power quickly receded below the control condition. In the $\gamma$ frequency band, the result revealed that part of the overall increase in power we observed under NB stimulation (Fig. 3) was associated with this phantom increase. 
A
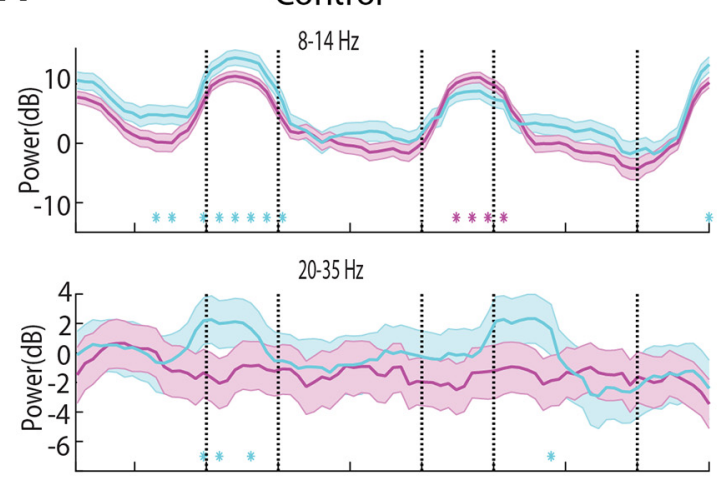

$45-100 \mathrm{~Hz}$

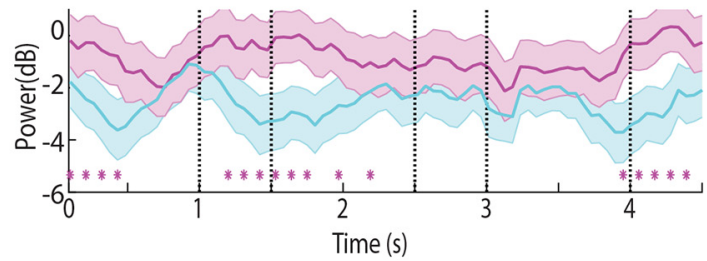

B
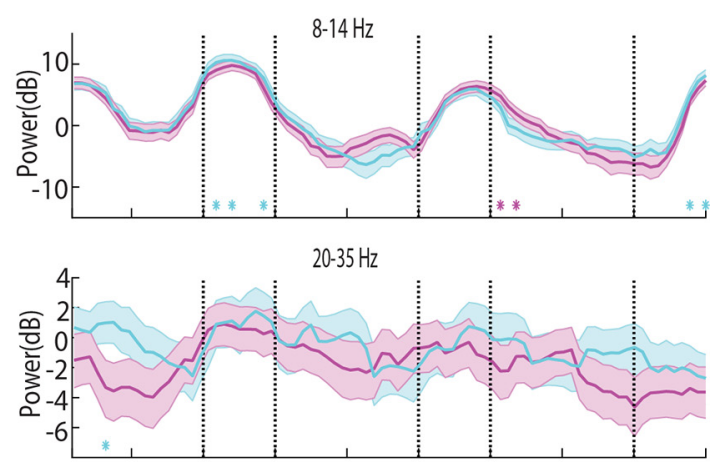

$45-100 \mathrm{~Hz}$

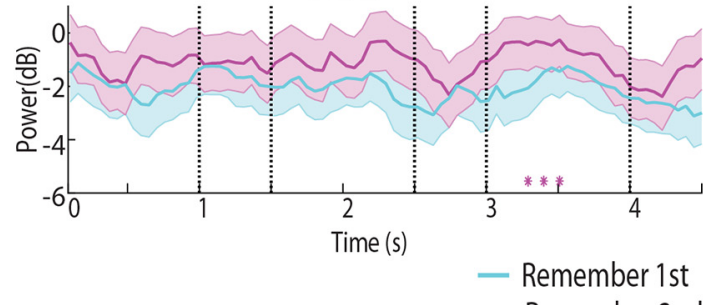

Figure 7. Time course of spectral power for remember first and remember second task. Average power is plotted for the same dataset as that shown in Figure 6. A, Time course of spectral power in the remember-first and remember-second tasks under control conditions. B, Spectral power under NB simulation. Stars represent time points where the difference between the two conditions plotted was found to be significant based on a permutation test, evaluated at the 0.01 significance level.

A
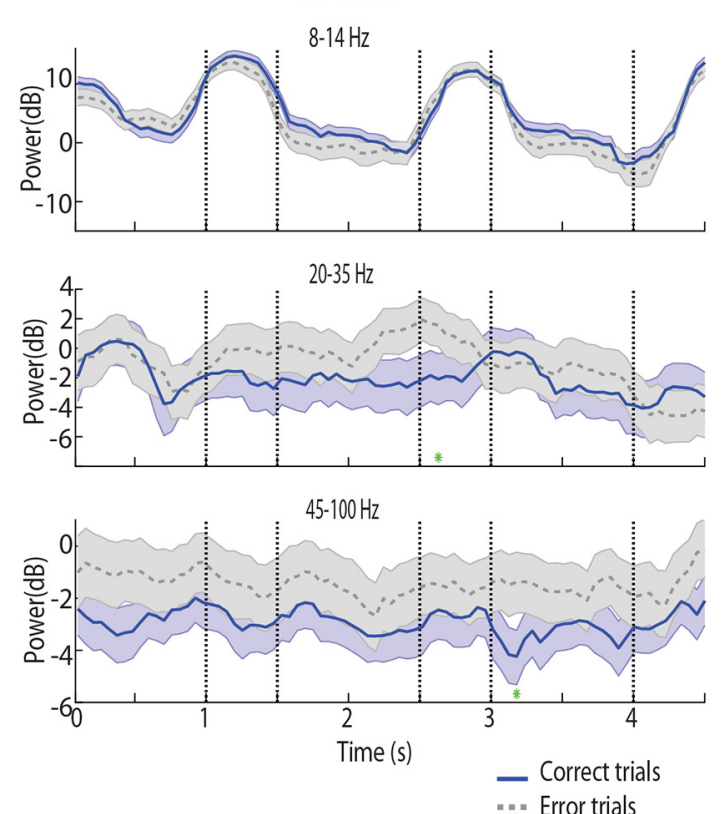

B
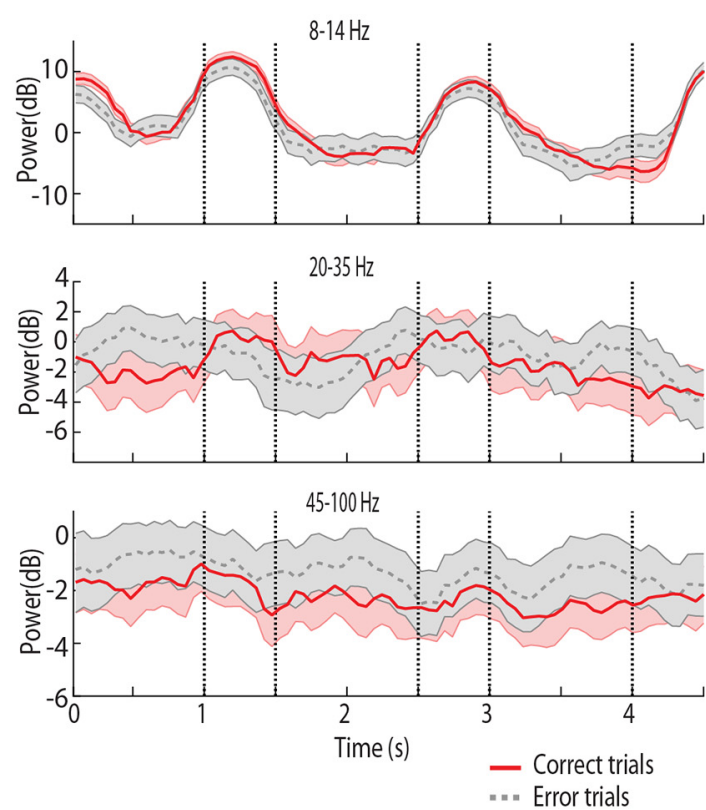

Figure 8. Power spectrum for correct and error trials. $\boldsymbol{A}$, Time course of spectral power recorded from the prefrontal cortex during the control condition, after subtracting the mean power of the baseline fixation period at each frequency band for correct $(n=7846$ trials) and error trials $(n=1987$ trials). $\boldsymbol{B}$, As in $\boldsymbol{A}$, under NB stimulation for correct $(n=6201$ trials) and error trials ( $n=1561$ trials). Stars represent time points where the difference between the two conditions plotted was found to be significant based on a permutation test, evaluated at the 0.01 significance level. 
A
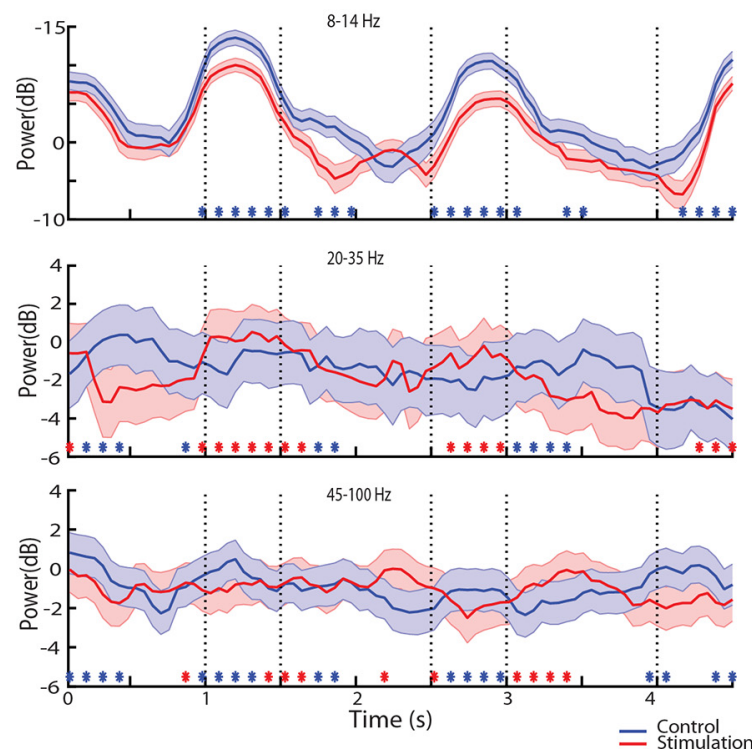

B
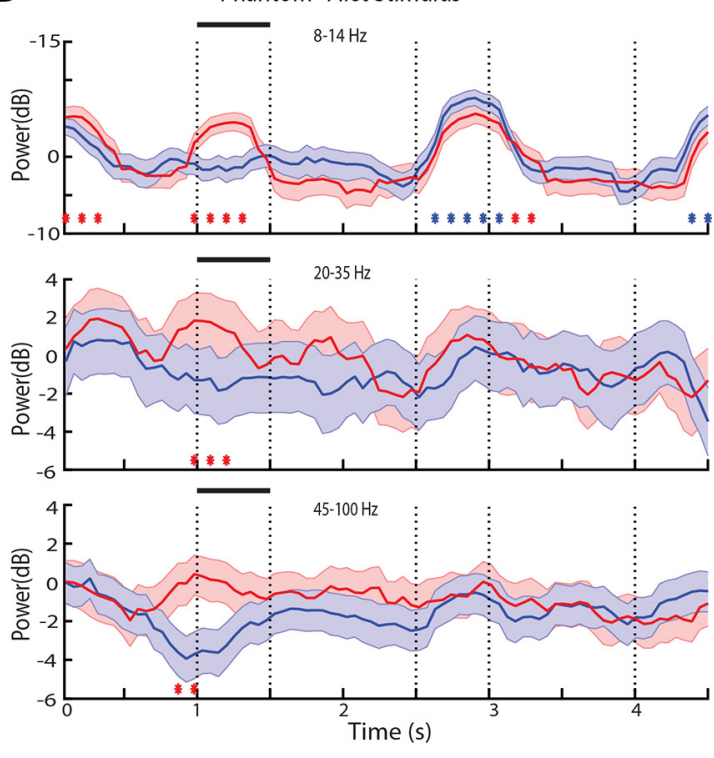

Figure 9. Power spectrum in catch trials. A, Time course of spectral power recorded from the prefrontal cortex during the control condition, after subtracting the mean power of the baseline fixation period at each frequency band for control ( $n=3334)$ and NB stimulation $(n=2629)$ trials. $\boldsymbol{B}$, As in $\boldsymbol{A}$, for trials in which the first stimulus was omitted $(n=884$ control and $n=613$ NB stimulation trials). Horizontal lines represent the expected time of the first stimulus presentation, which was missing in this condition.

\section{Discussion}

Our study documents the effects of intermittent NB stimulation on population measures of neuronal activity, as reflected in the LFP. Dysfunction or degeneration of the cholinergic forebrain is responsible for cognitive deficits in humans (Terry and Buccafusco, 2003; Ballinger et al., 2016), an effect that is reliably replicated by animal studies employing cholinergic antagonists or lesions (Chudasama et al., 2004; Croxson et al., 2011; Zhou et al., 2011). NB stimulation has been shown to be effective in improving performance in working memory as well as other cognitive tasks (Blake et al., 2017; Liu et al., 2017, 2018; Qi et al., 2021). As such, it holds promise for the treatment of cognitive decline in conditions such as age-related dementia and Alzheimer's disease (Subramaniam et al., 2021). However, substantial barriers remain before an effective translation of such an intervention to the clinic (Nazmuddin et al., 2021). Our results provide insights with direct relevance to optimizing the intervention, as they document signatures of rhythmic activity in cases where successful behavioral effects were documented. If similar changes in activity were to be observed in human patients, e.g., through frontal EEG readings, for stimulation sites that provided effective intervention, they could provide an independent means of verifying the effectiveness of the electrode placement, which is a non-trivial problem in clinical targeting (Subramaniam et al., 2021).

\section{Prefrontal activity mediating improvements in cognitive function}

The prefrontal cortex is an area critical for working memory and cognitive plasticity (Constantinidis and
Klingberg, 2016; Jaffe and Constantinidis, 2021). It receives innervation from a dedicated sub-region of the NB (Gielow and Záborszky, 2017) and thus is likely to play a role in the cognitive effects of stimulation. The mechanisms through which changes in prefrontal activity mediate improvement in working memory performance are less straightforward. Pharmacological studies suggest that cholinergic agonists increase neuronal activity specifically for the neuron's preferred stimulus, therefore sharpening its neuronal tuning neurons (Yang et al., 2013; Sun et al., 2017; Dasilva et al., 2019). Muscarinic and nicotinic- $\alpha 7$ inhibitors reduce prefrontal activity and abolishes tuning (Zhou et al., 2011; Yang et al., 2013; Major et al., 2015; Galvin et al., 2020). In contrast to these pharmacological results, NB stimulation revealed general increases in prefrontal activity but no enhancement of a neuron's preferred stimulus responses, and broadening rather than narrowing of tuning for stimuli (Qi et al., 2021). One possible explanation is that such broadening of prefrontal receptive fields serves to stabilize working memory and make it more resistant to distractors, at least in conditions for which fine discrimination between stimuli is not necessary (Qi et al., 2021).

However, this interpretation is predicated on mechanisms of working memory that depend on attractor models of working memory generation (Compte et al., 2000; Wimmer et al., 2014), a topic that is being hotly debated in the current literature (Constantinidis et al., 2018; Lundqvist et al., 2018). By other accounts, the rhythmicity of discharges is the critical neuronal variable that mediates working memory (Miller et al., 2018). Our current study revealed how NB stimulation altered rhythmicity of LFPs. 


\section{Changes in $\gamma$ power}

We directly tested whether $\gamma$ frequency power is increased under NB stimulation and whether this could account for improved working memory. LFP $\gamma$ power is known to be tuned to properties of stimuli held in memory, including the spatial location of the cue in the ODR task (Pesaran et al., 2002; Holmes et al., 2018). In rodents, GABAergic projections from the basal forebrain are electrically coupled and responsible for precisely-timed, rhythmic entrainment of cortical neurons, which is evident in increase of $\gamma$-band oscillations when they are selectively activated (McKenna et al., 2013; Kim et al., 2015). NB stimulation (unlike cholinergic pharmacological administration) is likely to activate such ascending GABAergic projections (Walker et al., 1989; Záborszky et al., 2018) and it therefore appeared plausible that NB stimulation might increase $\gamma$ power of LFPs. Contrary to these predictions, however, our present results show that the aggregate effect of NB stimulation did not increase LFP $\gamma$-band power in the prefrontal cortex during the delay periods of the task. An overall increase in $\gamma$ power was observed after NB stimulation, though this was present throughout the trial rather than the working memory intervals of the task, and its effects manifested themselves in different frequency bands for the two animals. $\gamma$ Power did exhibit differences between tasks in our experiment, being higher in the more demanding, remember-second task. However, NB stimulation tended to diminish rather than accentuate this difference. Recent results in the rodent auditory cortex did not detect increased $\gamma$ power after NB stimulation, suggesting that effects of cholinergic stimulation do not depend on $\gamma$ band increase (Azimi et al., 2020).

An important caveat for the interpretation of our results is the choice of baseline period. We relied on the intertrial interval as the baseline period for most analysis and power from this baseline was subtracted from all trials. Since the color of the fixation point in our experimental design was informative about the rule, we opted to use the inter-trial-interval as the baseline period, rather than the fixation interval. The absence of $\gamma$ power modulation we report therefore, refers specifically to additional increase in power during the delay period over the baseline, which did not differ between control and NB stimulation conditions. It is also possible that more subtle changes in $\gamma$ power occur during the maintenance of working memory, for example, shortlived $\gamma$-frequency "bursts" (Lundqvist et al., 2016). While we cannot rule out such bust events with the present analysis, their cumulative effect in the delay period was not visible across trials.

\section{Changes in $\alpha$ power}

Although it was not our a priori hypothesis, changes in the $\alpha$ band power proved the most sensitive indicator of different conditions in our experiment. $\alpha$ Power decreased by NB stimulation compared with control, particularly in the delay periods of the task, when the subjects need to maintain the relevant information in memory. $\alpha$ Power also differentiated the remember-first and remember-second tasks, being higher during the stimulus presentation period of whichever stimulus were to be maintained in memory. Similarly, $\alpha$ power was reduced in error trials compared with correct ones. Finally, NB stimulation produced a phantom increase in $\alpha$ power in periods over which a stimulus appearance was expected but did not occur.

$\alpha$ Power in EEG studies has been traditionally viewed as indicative of an idling state of cortex and elevated $\alpha$ power predictive of errors in processing because of lack of engagement in a task; however, more recently this notion has been shifted toward of a view that $\alpha$ power in sensory cortex reflects active suppression of task-irrelevant information (Klimesch, 2012; Clayton et al., 2015; Peylo et al., 2021). For example, when attention is shifted to color as opposed to motion in the context of a task, or to one hemifield over another, or to sensory modalities other than vision, $\alpha$ power increases in the cortical areas that are irrelevant for the task: the dorsal visual stream, ipsilateral hemisphere, or the visual cortex, respectively (Foxe et al., 1998; Snyder and Foxe, 2010; Doesburg et al., 2016). Increased entraining of $\alpha$ power by means of TMS rhythmic stimulation can bias visual perception in the ipsilateral visual field (Kasten et al., 2020). In the context of working memory, increased $\alpha$ power in posterior EEG electrodes is observed in tasks requiring suppression of distractors, and with increasing memory load (Hu et al., 2019).

Such a role of $\alpha$ band power is less clear in the frontal cortex, however. Reversed patterns of $\alpha$ rhythmicity between sensory and association cortex have been described in neurophysiological experiments in monkeys (Bollimunta et al., 2008). In the ODR task, $\alpha$ power is known to be elevated during the stimulus presentation, but does not otherwise appear tuned to stimulus properties in the delay period (Holmes et al., 2018). In our experiment, $\alpha$ power was indeed higher during the presentation of a stimulus that needed to be remembered (the first one in the rememberfirst task, and the second one in the remember-second task) and higher in correct than error trials. In this context, the decrease of $\alpha$ power by NB stimulation during the delay period would suggest that network is less likely to shift attention to the second stimulus. This interpretation is broadly in agreement with the analysis of behavioral and neurophysiological results (Qi et al., 2021): NB stimulation tended to stabilize the activity of prefrontal neurons and make memory of the stimulus less likely to shift (except in the case of nearby stimuli). Our current results suggest that these changes in attentional selection were more likely to be reflected in the $\alpha$ rather than the $\gamma$ frequency power.

\section{References}

Azimi H, Klaassen AL, Thomas K, Harvey MA, Rainer G (2020) Role of the thalamus in basal forebrain regulation of neural activity in the primary auditory cortex. Cereb Cortex 30:4481-4495.

Ballinger EC, Ananth M, Talmage DA, Role LW (2016) Basal forebrain cholinergic circuits and signaling in cognition and cognitive decline. Neuron 91:1199-1218.

Birks JS, Harvey RJ (2018) Donepezil for dementia due to Alzheimer's disease. Cochrane Database Syst Rev 6:CD001190.

Blake DT, Terry AV, Plagenhoef M, Constantinidis C, Liu R (2017) Potential for intermittent stimulation of nucleus basalis of Meynert to impact treatment of Alzheimer's disease. Commun Integr Biol 10:e1389359. 
Bokil H, Andrews P, Kulkarni JE, Mehta S, Mitra PP (2010) Chronux: a platform for analyzing neural signals. J Neurosci Methods 192:146-151.

Bollimunta A, Chen Y, Schroeder CE, Ding M (2008) Neuronal mechanisms of cortical alpha oscillations in awake-behaving macaques. J Neurosci 28:9976-9988.

Buzsaki G (2004) Large-scale recording of neuronal ensembles. Nat Neurosci 7:446-451.

Chudasama Y, Dalley JW, Nathwani F, Bouger P, Robbins TW, Nathwani $F$ (2004) Cholinergic modulation of visual attention and working memory: dissociable effects of basal forebrain 192-lgGsaporin lesions and intraprefrontal infusions of scopolamine. Learn Mem 11:78-86.

Clayton MS, Yeung N, Cohen Kadosh R (2015) The roles of cortical oscillations in sustained attention. Trends Cogn Sci 19:188-195.

Compte A, Brunel N, Goldman-Rakic PS, Wang XJ (2000) Synaptic mechanisms and network dynamics underlying spatial working memory in a cortical network model. Cereb Cortex 10:910-923.

Constantinidis C, Klingberg T (2016) The neuroscience of working memory capacity and training. Nat Rev Neurosci 17:438-449.

Constantinidis C, Funahashi S, Lee D, Murray JD, Qi XL, Wang M, Arnsten AFT (2018) Persistent spiking activity underlies working memory. J Neurosci 38:7020-7028.

Croxson PL, Kyriazis DA, Baxter MG (2011) Cholinergic modulation of a specific memory function of prefrontal cortex. Nat Neurosci 14:1510-1512.

Dasilva M, Brandt C, Gotthardt S, Gieselmann MA, Distler C, Thiele A (2019) Cell class-specific modulation of attentional signals by acetylcholine in macaque frontal eye field. Proc Natl Acad Sci USA 116:20180-20189.

Doesburg SM, Bedo N, Ward LM (2016) Top-down alpha oscillatory network interactions during visuospatial attention orienting. Neuroimage 132:512-519.

Foxe JJ, Simpson GV, Ahlfors SP (1998) Parieto-occipital approximately $10 \mathrm{~Hz}$ activity reflects anticipatory state of visual attention mechanisms. Neuroreport 9:3929-3933.

Freund HJ, Kuhn J, Lenartz D, Mai JK, Schnell T, Klosterkoetter J, Sturm V (2009) Cognitive functions in a patient with Parkinson-dementia syndrome undergoing deep brain stimulation. Arch Neurol 66:781-785

Fries $P$ (2009) Neuronal gamma-band synchronization as a fundamental process in cortical computation. Annu Rev Neurosci 32:209-224.

Galvin VC, Arnsten AFT, Wang M (2018) Evolution in neuromodulation-the differential roles of acetylcholine in higher order association vs. primary visual cortices. Front Neural Circuits 12:67.

Galvin VC, Yang ST, Paspalas CD, Yang Y, Jin LE, Datta D, Morozov YM, Lightbourne TC, Lowet AS, Rakic P, Arnsten AFT, Wang M (2020) Muscarinic M1 receptors modulate working memory performance and activity via $\mathrm{KCNQ}$ potassium channels in the primate prefrontal cortex. Neuron 106:649-661.e4. Epub ahead of print.

Gielow MR, Záborszky L (2017) The input-output relationship of the cholinergic basal forebrain. Cell Rep 18:1817-1830.

Hardenacke K, Hashemiyoon R, Visser-Vandewalle V, Zapf A, Freund HJ, Sturm V, Hellmich M, Kuhn J (2016) Deep brain stimulation of the nucleus basalis of Meynert in Alzheimer's dementia: potential predictors of cognitive change and results of a long-term follow-up in eight patients. Brain Stimul 9:799-800.

Holmes CD, Papadimitriou C, Snyder LH (2018) Dissociation of LFP power and tuning in the frontal cortex during memory. J Neurosci 38:8177-8186.

Hu Z, Barkley CM, Marino SE, Wang C, Rajan A, Bo K, Samuel IBH, Ding M (2019) Working memory capacity is negatively associated with memory load modulation of alpha oscillations in retention of verbal working memory. J Cogn Neurosci 31:1933-1945.

Jaffe RJ, Constantinidis C (2021) Working memory: from neural activity to the sentient mind. Compr Physiol 11:1-41.

Kajikawa Y, Schroeder CE (2011) How local is the local field potential? Neuron 72:847-858.
Kasten FH, Wendeln T, Stecher HI, Herrmann CS (2020) Hemisphere-specific, differential effects of lateralized, occipitalparietal $\alpha$-versus $\gamma$-tACS on endogenous but not exogenous visual-spatial attention. Sci Rep 10:12270.

Kim T, Thankachan S, McKenna JT, McNally JM, Yang C, Choi JH, Chen L, Kocsis B, Deisseroth K, Strecker RE, Basheer R, Brown RE, McCarley RW (2015) Cortically projecting basal forebrain parvalbumin neurons regulate cortical gamma band oscillations. Proc Natl Acad Sci USA 112:3535-3540.

Klimesch W (2012) $\alpha$-Band oscillations, attention, and controlled access to stored information. Trends Cogn Sci 16:606-617.

Kuhn J, Hardenacke K, Lenartz D, Gruendler T, Ullsperger M, Bartsch C, Mai JK, Zilles K, Bauer A, Matusch A, Schulz RJ, Noreik M, Bührle CP, Maintz D, Woopen C, Häussermann P, Hellmich M, Klosterkötter J, Wiltfang J, Maarouf M, et al. (2015) Deep brain stimulation of the nucleus basalis of Meynert in Alzheimer's dementia. Mol Psychiatry 20:353-360.

Liu R, Crawford J, Callahan PM, Terry AV Jr, Constantinidis C, Blake DT (2017) Intermittent stimulation of the nucleus basalis of Meynert improves working memory in adult monkeys. Curr Biol 27:2640-2646.

Liu R, Crawford J, Callahan PM, Terry AV, Constantinidis C, Blake DT (2018) Intermittent stimulation in the nucleus basalis of meynert improves sustained attention in rhesus monkeys. Neuropharmacology 137:202-210.

Lundqvist M, Rose J, Herman P, Brincat SL, Buschman TJ, Miller EK (2016) Gamma and beta bursts underlie working memory. Neuron 90:152-164.

Lundqvist M, Herman P, Miller EK (2018) Working memory: delay activity, yes! Persistent activity? Maybe not. J Neurosci 38:70137019.

Major AJ, Vijayraghavan S, Everling S (2015) Muscarinic attenuation of mnemonic rule representation in macaque dorsolateral prefrontal cortex during a pro- and anti-saccade task. J Neurosci 35:16064-16076.

Maris E, Oostenveld R (2007) Nonparametric statistical testing of EEG- and MEG-data. J Neurosci Methods 164:177-190.

McCairn KW, Turner RS (2009) Deep brain stimulation of the globus pallidus internus in the parkinsonian primate: local entrainment and suppression of low-frequency oscillations. J Neurophysiol 101:1941-1960.

McKenna JT, Yang C, Franciosi S, Winston S, Abarr KK, Rigby MS, Yanagawa Y, McCarley RW, Brown RE (2013) Distribution and intrinsic membrane properties of basal forebrain GABAergic and parvalbumin neurons in the mouse. J Comp Neurol 521:12251250.

Mesulam MM, Mufson EJ, Levey Al, Wainer BH (1983) Cholinergic innervation of cortex by the basal forebrain: cytochemistry and cortical connections of the septal area, diagonal band nuclei, nucleus basalis (substantia innominata), and hypothalamus in the rhesus monkey. J Comp Neurol 214:170-197.

Meyer T, Constantinidis C (2005) A software solution for the control of visual behavioral experimentation. J Neurosci Methods 142:2734.

Miller EK, Lundqvist M, Bastos AM (2018) Working memory 2.0. Neuron 100:463-475.

Nazmuddin M, Philippens I, van Laar T (2021) Electrical stimulation of the nucleus basalis of Meynert: a systematic review of preclinical and clinical data. Sci Rep 11:11751.

Oostenveld R, Fries P, Maris E, Schoffelen JM (2011) FieldTrip: open source software for advanced analysis of MEG, EEG, and invasive electrophysiological data. Comput Intell Neurosci 2011:156869.

Pesaran B, Pezaris JS, Sahani M, Mitra PP, Andersen RA (2002) Temporal structure in neuronal activity during working memory in macaque parietal cortex. Nat Neurosci 5:805-811.

Peylo C, Hilla Y, Sauseng P (2021) Cause or consequence? Alpha oscillations in visuospatial attention. Trends Neurosci 44:705-713.

Qi XL, Liu R, Singh B, Bestue D, Compte A, Vazdarjanova Al, Blake DT, Constantinidis C (2021) Nucleus basalis stimulation enhances 
working memory by stabilizing stimulus representations in primate prefrontal cortical activity. Cell Rep 36:109469.

Riley MR, Qi XL, Constantinidis C (2017) Functional specialization of areas along the anterior-posterior axis of the primate prefrontal cortex. Cereb Cortex 27:3683-3697.

Roux F, Uhlhaas PJ (2014) Working memory and neural oscillations: $\alpha-\gamma$ versus $\theta-\gamma$ codes for distinct WM information? Trends Cogn Sci 18:16-25.

Sarter M, Lustig C, Berry AS, Gritton H, Howe WM, Parikh V (2016) What do phasic cholinergic signals do? Neurobiol Learn Mem 130:135-141.

Snyder AC, Foxe JJ (2010) Anticipatory attentional suppression of visual features indexed by oscillatory alpha-band power increases: a high-density electrical mapping study. J Neurosci 30:40244032.

Subramaniam S, Blake DT, Constantinidis C (2021) Cholinergic deep brain stimulation for memory and cognitive disorders. J Alzheimers Dis 83:491-503.

Sun Y, Yang Y, Galvin VC, Yang S, Arnsten AF, Wang M (2017) Nicotinic $\alpha 4 \beta 2$ cholinergic receptor influences on dorsolateral prefrontal cortical neuronal firing during a working memory task. $J$ Neurosci 37:5366-5377.

Terry AV Jr, Buccafusco JJ (2003) The cholinergic hypothesis of age and Alzheimer's disease-related cognitive deficits: recent challenges and their implications for novel drug development. J Pharmacol Exp Ther 306:821-827.

Uhlhaas PJ, Singer W (2011) The development of neural synchrony and large-scale cortical networks during adolescence: relevance for the pathophysiology of schizophrenia and neurodevelopmental hypothesis. Schizophr Bull 37:514-523.

Walker LC, Price DL, Young WS 3rd (1989) GABAergic neurons in the primate basal forebrain magnocellular complex. Brain Res 499:188-192.

Wimmer K, Nykamp DQ, Constantinidis C, Compte A (2014) Bump attractor dynamics in prefrontal cortex explains behavioral precision in spatial working memory. Nat Neurosci 17:431-439.

Yang Y, Paspalas CD, Jin LE, Picciotto MR, Arnsten AF, Wang M (2013) Nicotinic $\alpha 7$ receptors enhance NMDA cognitive circuits in dorsolateral prefrontal cortex. Proc Natl Acad Sci USA 110:1207812083.

Záborszky L, Gombkoto P, Varsanyi P, Gielow MR, Poe G, Role LW, Ananth M, Rajebhosale P, Talmage DA, Hasselmo ME, Dannenberg $H$, Minces VH, Chiba AA (2018) Specific basal forebrain-cortical cholinergic circuits coordinate cognitive operations. J Neurosci 38:94469458.

Zhou X, Qi XL, Douglas K, Palaninathan K, Kang HS, Buccafusco JJ, Blake DT, Constantinidis C (2011) Cholinergic modulation of working memory activity in primate prefrontal cortex. J Neurophysiol 106:2180-2188. 